\title{
Annual Cycle of Surface Longwave Radiation
}

\author{
Pamela E. Mlynczak ${ }^{1}$, G. Louis Smith ${ }^{2}$, Anne C. Wilber ${ }^{1}$ and Paul W. Stackhouse ${ }^{3}$ \\ 1. SSAI, Hampton, Virginia \\ 2. National Institute of Aerospace, Hampton, Virginia \\ 3. Langley Research Center, Hampton, Virginia
}

Corresponding author address: G. Louis Smith, NASA Langley Research Center, Mail Stop 420, Hampton, Virginia 23681-2199.

E-mail: george.1.smith@nasa.gov 


\begin{abstract}
The annual cycles of upward and downward longwave fluxes at the Earth's surface are investigated by use of the NASA/GEWEX Surface Radiation Budget Data Set. Because of the immense difference between the heat capacity of land and ocean, the surface of Earth is partitioned into these two categories. Principal component analysis is used to quantify the annual cycles. Over land, the first principal component describes over $95 \%$ of the variance of the annual cycle of the upward and downward longwave fluxes. Over ocean the first term describes more than $87 \%$ of these annual cycles. Empirical orthogonal functions show the corresponding geographical distributions of these cycles. Phase plane diagrams of the annual cycles of upward longwave fluxes as a function of net shortwave flux show the thermal inertia of land and ocean.
\end{abstract}




\section{Introduction}

Fluxes of radiation at the surface of the Earth are intimately related to climate. The weather/climate system is a heat engine for which the source of heat is the Sun's radiation. Most of the radiation from the Sun which is absorbed by the Earth-atmosphere system is absorbed at the surface. The longwave radiation flux emitted by the surface and the longwave flux from the atmosphere down to the surface both exceed the radiation absorbed from the Sun. An understanding of the Earth's climate must include knowledge of the surface radiation budget as it varies geographically and temporally (Ramanathan 1986; WCRP-69 1992). There are two major cycles of solar forcing of the weather and climate system: the diurnal cycle and the annual cycle. These two cycles have time scales two orders of magnitude apart, and the relevant processes are quite different. The diurnal cycle is short enough that most processes involved are local and tend to be in the planetary boundary layer, unless the heating is sufficient to develop convection. The annual cycle is long enough that the important processes are global in scale.

The annual cycles of atmospheric and oceanic processes are well-known, and the annual cycles of surface temperature, pressure and precipitation have been quantified by several researchers, e.g. White and Wallace (1978), Hsu and Wallace (1976a, b). The annual cycle of outgoing longwave radiation at the top of the atmosphere was studied by Bess et al. (1992) using data from the Earth Radiation Budget (ERB) instruments aboard the Nimbus-6 and -7 spacecraft. Smith et al. (1990) investigated the annual cycle of absorbed solar radiation at the top of the atmosphere using data from these two instruments. Hatzianastassiou et al. (2004) investigated the net longwave, net shortwave, and net total radiation at the top of the atmosphere and at the surface over a ten-year period from 1984 to 1993. They reported the global distribution of surface radiation for the annual mean values and for the January, April, July and October 
monthly means. They extended their surface shortwave radiation budget study to a 17 -year period in Hatzianastassiou et al. (2005).

A Surface Radiation Budget (SRB) data set was initiated at Langley Research Center in support of the World Climate Research Program (Whitlock et al. 1995). This data set used cloud and radiance data from the International Satellite Cloud Climatology Project (ISCCP; Rossow and Schiffer 1991) with an algorithm by Darnell et al. (1988) to compute surface shortwave fluxes and an algorithm by Gupta (1989) to compute longwave surface fluxes. Darnell et al. (1992) examined the four midseasonal months based on the first year of data processed. They presented maps of surface radiation components and latitudinal plots of zonal averages of the components of surface radiation. Gupta et al. (1993) investigated the cloud forcing for upward and downward shortwave, longwave and total radiation fluxes for July 1985 and January 1986. The SRB data set was extended and improved, and Gupta et al. (1999) reported further on the climatology of SRB. They described the annual cycles of zonal means by time-latitude plots for downward and net shortwave and longwave fluxes. Wilber et al. (2006) used a later version of the SRB data set to study the annual cycle of solar radiation at the surface by use of principal component analysis.

The SRB data set has been upgraded further as part of the Global Energy and Water Experiment (GEWEX; Stackhouse et al. 2004; Cox et al. 2006; Gupta et al. 2006) and is now designated the NASA/GEWEX SRB Release 3.0 Data Set. It includes upward and downward shortwave and longwave radiation fluxes at the surface with a $1^{\circ} \times 1^{\circ}$ grid. This data base permits the study of the spatial distribution of surface radiation components during the period 1983-2007.

The present paper examines the SRB data set to investigate the time and geographical variations of longwave radiation components at the surface, i.e., the upward longwave flux 
LWU, the downward longwave flux LWD (from the atmosphere) and the net longwave flux LWN. The investigation uses a principal component analysis, whereby the major cyclic effects are computed as time variations with maps revealing their geographical distributions. The advantage of this approach is that it represents the time and space variations with the minimum number of terms, which are determined by the data. The principal components are statistical descriptors rather than physical, but they often have simple physical interpretations. Climatological relations among the annual cycles of net shortwave flux SWN, LWU and LWD are studied in terms of their principal components.

\section{Data Set}

The NASA/GEWEX Surface Radiation Budget Release 3.0 Data Set (Cox et al., 2006; Gupta et al., 2006) was used for this study. This data set uses International Satellite Cloud Climatology Project (ISCCP) clouds and radiances and other inputs to produce monthly-mean upward and downward longwave and shortwave radiative fluxes on a $1^{\circ} \times 1^{\circ}$ grid.

The current Release 3.0 covers the period from July 1983 through December 2007. Shortwave fluxes are computed using an algorithm adapted from Pinker and Laszlo (1992) which converts narrowband radiances from ISCCP to fluxes at the top of the atmosphere using Earth Radiation Budget Experiment (ERBE) bidirectional models and then uses a table lookup based on a delta-Eddington two stream computation. Longwave fluxes are computed with an adaptation of the Fu et al. (1997) method. Cloud properties are derived from ISCCP DX data (Rossow and Schiffer 1999). Temperature and humidity profiles are taken from the Goddard Earth Observing System Data Assimilation System, level-4 (GEOS-4) at six-hourly intervals starting at midnight UTC (Bloom et al. 2005); other surface parameters are available from 
GEOS-4 on three-hourly intervals. For cloud cover less than $50 \%$, the skin temperature from ISCCP is used for computing longwave flux from the surface, and for cloud cover greater than $50 \%$, the surface temperature is taken from GEOS- 4 . Where ice or snow cover exceeds $80 \%$, the ISCCP skin temperature is used. The ocean temperatures in GEOS-4 are linearly interpolated from the Reynolds' weekly mean SST analysis.

\section{Analysis Method}

There are 44016 regions in the nested grid of the data set, each with monthly-mean values for each parameter. These are regridded onto a $360^{\circ} \times 180^{\circ}$ equal angle grid using replication. The first step in the analysis is to compute the mean value of each flux (LWU, LWD and LWN) over all 24 Januaries to form an average January for the period from 1983 through 2007 for each $1^{\circ} \times 1^{\circ}$ grid box, or region, and likewise for every month. Next, the annual average is computed and subtracted from the value for each month. Thus, a flux may be expressed as

$$
F(\tau, x)=\operatorname{Mean}(x)+A(\tau, x)
$$

where $A(\tau, x)$ is the annual cycle for month $\tau$ and location $x$.

In order to examine the annual cycle of all 64800 regions both qualitatively and quantitatively, principal component analysis (PCA) is used. This method has the advantage that the first PC describes the maximum amount of variance of the annual cycle in time and space that is possible with one function, and each successive PC explains the maximum amount of variance of the residual (Smith et al. 2008). Because of the enormous thermal inertia of the ocean, the annual cycles of longwave fluxes over the ocean are much smaller than over land; thus land and ocean regions are treated separately, while regions with mixed land and ocean are excluded. First, a $12 \times 12$ covariance matrix is computed as 


$$
\mathbf{M}=\sum_{x} w(x) A(\tau, x) A\left(\tau^{\prime}, x\right)
$$

where $A(\tau, x)$ denotes the annual cycle for month $\tau$ and region $x, w(x)$ is the area weighting for the $x^{\text {th }}$ region, and the summation is over all land or ocean regions. The eigenvalues $\lambda_{i}$ and the eigenvectors, namely the principal components $P C_{i}(\tau)$, of this covariance matrix are then computed. The PCs are orthogonal and provide a useful basis set for describing the time variation of the annual cycles. Finally the empirical orthogonal functions $E O F_{i}(x)$, which

describe the geographical variation of the annual cycles, are computed by projecting the 12 $P C_{i}(\tau)$ onto the 12 monthly maps for each parameter. The annual cycle of a parameter at region $x$ is thus given by

$$
A(\tau, x)=\sum_{i} P C_{i}(\tau) E O F_{i}(x)
$$

The PCs and EOFs provide a description that is useful to the extent that one can physically interpret them. The physical interpretation depends on the shape of the PC, the geographical location of the large EOF values, and recognition of the processes that occur in these regions. The interpretation is thus subjective to some degree.

\section{Time and Space Variations}

The annual cycle of radiation is taken to be the variation of the radiation about its annual mean. The global mean values of the surface radiation fluxes are first presented, then the geographic distributions of annual average fluxes are discussed. The annual cycles are described next. After the time and space variations of the annual cycles are shown, the relations between the surface radiation fluxes are examined. 


\section{a. Global-mean surface radiation fluxes}

The global mean surface radiation fluxes are summarized in Table 1. The shortwave net SWN is $166.5 \mathrm{~W} \mathrm{~m}^{-2}$, longwave flux up LWU is $396.4 \mathrm{~W} \mathrm{~m}^{-2}$, and the longwave flux down LWD is $343.8 \mathrm{~W} \mathrm{~m}^{-2}$. The difference, longwave net $\mathrm{LWN}$, is $-52.6 \mathrm{~W} \mathrm{~m}^{-2}$. For comparison, Hatzianastassiou et al. (2004) reported the global annual mean of LWN to be a cooling of 49.7 $\mathrm{W} \mathrm{m}^{-2}$ and of SWN to be $147.3 \mathrm{~W} \mathrm{~m}^{-2}$. With an extended time period, Hatzianastassiou et al. (2005) computed the global annual mean of SWN to be $149.4 \mathrm{~W} \mathrm{~m}^{-2}$. The ratio of the globalaverage annual-mean values of LWD to LWU is 0.87 .

Table 1 also shows the mean values of the fluxes averaged over land and over ocean. Grid boxes that contain a mix of ocean and land are not included and constitute the missing $6.3 \%$ of the surface area.

\section{b. Annual mean longwave fluxes}

Figure 1 shows global maps of annual mean surface fluxes of LWU, LWD and LWN. The annual mean LWU was computed using monthly means from the SRB data set and is shown by Fig. 1a. LWU has a strongly zonal appearance, as does the SWN (Wilber et al. 2006), which drives LWU. The meridional variations are primarily due to clouds and to a smaller degree, aerosols. The clouds over the Congo Basin and the eastern oceans decrease the SWN, reducing the surface temperature and LWU. The temperature of the eastern oceans is reduced by the currents from higher latitudes toward the Equator, decreasing LWU from its zonal mean. Over

the deserts of Northern Africa and the Arabian Peninsula, LWU exceeds $500 \mathrm{~W} \mathrm{~m}^{-2}$ due to low latitude and low cloud amounts that result in high SWN. 
Figure $1 \mathrm{~b}$ shows the annual mean LWD. The patterns are very similar to those of LWU, showing the close coupling of the atmosphere with the surface. LWN is the difference between LWU and LWD and is much smaller than LWU or LWD over most of the Earth, implying that the LWU and LWD maps must resemble each other closely.

Figure 1c shows the annual average LWN. The annual average LWN is negative over the entire planet, i.e. cooling. During winter night of polar regions, LWN becomes positive, that is the atmosphere heats the surface radiatively, but the average over the year is still negative. The low LWN over ocean indicates that the surface and lower troposphere are closely coupled by planetary boundary layer processes. Over most of the oceans with latitude less than $60^{\circ}$ in each hemisphere, LWN is between -40 and $-60 \mathrm{~W} \mathrm{~m}^{-2}$. Poleward of $60^{\circ}$ for most regions LWN is between -20 and $-40 \mathrm{~W} \mathrm{~m}^{-2}$ with a few regions between $-20 \mathrm{~W} \mathrm{~m}^{-2}$ and 0 . Over land regions with much cloud the magnitude of LWN cooling is less than $60 \mathrm{~W} \mathrm{~m}^{-2}$, and regions with small cloud amounts have cooling greater than $60 \mathrm{~W} \mathrm{~m}^{-2}$. LWN cooling is small over tropical wet climate regions. Over deserts, LWN cooling increases to 120 to $160 \mathrm{~W} \mathrm{~m}^{-2}$, with surrounding steppe regions having LWN cooling between 60 and $120 \mathrm{~W} \mathrm{~m}^{-2}$ (Smith et al. 2002). The dry descending air over deserts and steppes has a lesser opacity than the humid air over oceans and thus radiates less downward.

Figure 2 shows the zonal means of the radiation fluxes at the surface. In Fig. 2 the zonal mean SWN varies from $40 \mathrm{~W} \mathrm{~m}^{-2}$ near the poles to $215 \mathrm{~W} \mathrm{~m}^{-2}$ at the Equator, with a dip at $8^{\circ} \mathrm{N}$ due to the Intertropical Convergence Zone. The zonal mean LWU varies from $140 \mathrm{~W} \mathrm{~m}^{-2}$ at the South Pole to $460 \mathrm{~W} \mathrm{~m}^{-2}$ near the Equator to $210 \mathrm{~W} \mathrm{~m}^{-2}$ at the North Pole, so that the LWU in the Northern Hemisphere is higher than in the Southern Hemisphere. The zonal mean LWD has a shape similar to that of LWU. The dotted line is the zonal-mean LWU times the ratio of the 
global-mean LWD to LWU (0.87). The match between LWD and 0.87 LWU is within $2 \%$ with three exceptions. Near $20^{\circ} \mathrm{N}$, LWD is lower compared to the 0.87 LWU line due to the dry air over the deserts of North Africa and the Middle East, and near the Equator, LWD is higher due to the deep convection centers of the Amazon and Congo Basins and the Maritime Continent of the Philippines region. LWD is slightly lower near $30^{\circ} \mathrm{S}$ because of the deserts of the Southern Hemisphere. This simple relation between zonal averages of annual-mean LWD and LWU happens because of averaging over the year and around all longitudes, which eliminates regional and temporal variations.

Figure 3 shows the zonal mean LWN, which ranges between -20 and $-75 \mathrm{~W} \mathrm{~m}^{-2}$. As the difference between two large numbers, LWN varies much within its range. The minima are due to the deserts near $25^{\circ}$ in each hemisphere, and there is a local maximum at the Equator.

\section{c. Annual cycles of surface radiation}

Land and ocean have such different responses to the annual cycle of insolation that it is useful to separate them for study. The root-mean-square RMS of the differences of the monthly means from the annual mean values provides a measure over the domain of the magnitude of the annual cycle. Table 1 lists the global RMS values for the surface radiation fluxes. Over land the RMS of LWU is $46 \mathrm{~W} \mathrm{~m}^{-2}$, which is close to that for SWN. Because of the oceans' immense heat capacity, the RMS of LWU over ocean is only $13 \mathrm{~W} \mathrm{~m}^{-2}$. Over land, the RMS of LWD is smaller than LWU, but the two are essentially equal for ocean. For both cases the RMS of LWN is small compared to that for either LWU or LWD. 


\section{d. Annual cycles of surface radiation over land}

The annual cycles are described by use of principal components (PCs) for the time variations. Table 2 lists the first four normalized eigenvalues from the PCA. The first PCs for LWU and LWD describe more than $95 \%$ of the variance of the annual cycle. For LWN, PC-1 describes $81 \%$ of the variance, and PC-2 describes $10 \%$.

Figure 4 shows the annual cycles of the first four principal components for LWU over land. The first PC is an annual sine wave that peaks in July with $65 \mathrm{~W} \mathrm{~m}^{-2}$ and has a minimum of -60 $\mathrm{W} \mathrm{m} \mathrm{m}^{-2}$ in January. The RMS of each PC is the RMS of LWU times the square root of its associated eigenvalue. The first eigenvalue for LWU is 0.953 , so that the RMS of the PC-1 is $44.9 \mathrm{~W} \mathrm{~m}^{-2}$. If the PC is a sine wave, its amplitude is the RMS of the function times $2^{1 / 2}$, or 63.5 $\mathrm{W} \mathrm{m} \mathrm{m}^{-2}$.

PC-2 is a combination of a semiannual wave and an annual wave and describes $2.5 \%$ of the variance of LWU. The two minima of PC-2 are near the zeros of PC-1. The effect of PC-2 is to slow (where EOF-2 is positive) or speed up (where EOF-2 is negative) the increase of LWU in April. EOF-2 (not shown) has negative values over zones of North Africa and India that are related to the monsoons. PC-3 plays a similar role in speeding the decrease of LWU where EOF3 is positive.

The principal components for LWD for land are shown by Fig. 5. PC-1 is an annual cycle with its peak in July and minimum in December, in phase with PC-1 of LWU. Its maximum is 52 $\mathrm{W} \mathrm{m}^{-2}$, slightly smaller than for LWU. PC-2 describes only $1.8 \%$ of the variance for LWD and is a semiannual cycle. As with LWU, the minima for PC-2 are near the zeros for PC-1.

Figure 6 shows the first four principal components of LWN for land. As the difference between two approximately annual cycles, LWN PC-1 is also an annual cycle with maximum in 
July. However, PC-1 for LWN deviates somewhat from being a sine wave. PC-2 describes $10 \%$ of the variance of LWN and is largely an annual cycle with a small semiannual cycle added. The annual cycle portion of PC-2 is out of phase with PC-1, with its peak in April, near the zero of PC-1.

The PCs are projected onto the data set to produce their spatial counterparts, the empirical orthogonal functions, or EOF maps. Figure 7 shows maps of the first empirical orthogonal function EOF-1 for LWU, LWD and LWN, corresponding to the annual cycle. EOF-1 for LWU, shown in Fig. 7a, is zonal outside the Tropics, with a more complex pattern over the equatorial deep convection regions of the Amazon, the Congo, and the Maritime Continent of the Philippines as well as the monsoonal regions of South Asia. The largest values are across Asia and the Middle East. The null line, where EOF-1 equals zero, is about $10^{\circ}$ north of the Equator. The lower values over Greenland show that the LWU annual cycle there has a lower variation than in other high latitude regions.

The geographical pattern of LWD is shown by Fig. $7 \mathrm{~b}$. There is a basic increase with latitude, but with much longitudinal variation. The western parts of North America and Europe have lower values than regions eastward, where the moderating influence of the oceans has diminished and atmospheric temperature variations are greater. As with LWU, Greenland has a lower variation of PC-1 for LWD than do other high latitude regions.

The geographical distribution of EOF-1 for LWN is shown by Fig. 7c. The most noticeable feature of this plot is the reversal of latitudinal variation outside the Tropics from LWD and LWU. This reversal occurs because LWN is the difference between LWD and LWU, which has the greater amplitude. Again, there is complex longitudinal distribution, especially in the Middle East and South Asia regions, primarily due to the movement of cloud systems. 


\section{e. Annual cycles of surface radiation over ocean}

The eigenvalues for the principal components for longwave fluxes over ocean are listed in Table 3. The first four principal components for LWU over ocean are shown in Fig. 8. PC-1 for LWU accounts for $87 \%$ of the variance and is an annual cycle with an amplitude of $18 \mathrm{~W} \mathrm{~m}^{-2}$, less than one third that over land. Its maximum is in August, and its minimum is in February. The effects of heat storage have been to attenuate the annual cycle and to create a phase lag of one month after SWN. The PC-1 for LWD describes $90.5 \%$ of the variance and is seen in Fig. 9 to be an annual cycle. Its amplitude is $20 \mathrm{~W} \mathrm{~m}^{-2}$, and it also has extrema in August and February, indicating that the atmosphere is very closely coupled to the ocean surface. Figure 10 shows the principal components of LWN. For this case, PC-1 accounts for $71 \%$ of the variance. PC-1 ranges between -6 and $8 \mathrm{~W} \mathrm{~m}^{-2}$ and is an annual cycle.

Figure 11 shows the EOF-1 maps for LWU, LWD and LWN over ocean. For LWU and LWD, EOF-1 is between 0 and -1 standard deviation over the Southern Ocean except near Antarctica. The high latitude oceans are more variable, as continents affect the temperature of the ocean and the overlying atmosphere, creating greater variations of the annual cycle. Thus the color-bar covers \pm 4 standard deviations as compared to that for land, for which \pm 2 is sufficient in Fig. 7. However, the PC-1 for ocean has a much smaller amplitude than for land, so that the magnitudes of the annual cycles of LWU and LWD are not great. The LWN is the difference of the LWU and LWD, which are close; thus LWN has a complex structure. Although PC-1 for

LWN leaves $29 \%$ for higher order terms, the RMS of the remaining terms is only $3.0 \mathrm{~W} \mathrm{~m}^{-2}$ and is small compared to that for the higher order terms for LWU and LWD. 
Some of the effects of clouds on the longwave fluxes, up, down and net, for land and ocean have been discussed here. Wilber et al. (2006) described some of the effects of cloud on shortwave radiation. The quantitative evaluation of cloud forcing on the annual cycle of surface radiation budget must be reported in another paper.

\section{Relations among Radiation Components}

It has been shown that the preponderance of the variance of the annual cycles over land of SWN (Wilber et al. 2006), LWU and LWD is described by the first principal component in each case (more than 90\%). It is thus useful to examine the relations between these three terms. Figure 12 shows PC-1 for SWN, LWU and LWD. The SWN drives the surface radiation budget in most cases, heating the surface, which results in the LWU changing, with a phase lag due to heat storage. The LWD response lags the LWD slightly. Figure 13 shows the annual cycle of PC-1 of LWU as a function of PC-1 of SWN. The cycles of PC-1 for LWU and for SWN describe an ellipse. The ranges of both variables are about $\pm 65 \mathrm{~W} \mathrm{~m}^{-2}$, so that the major axis of the ellipse has a slope of one. The minimum and maximum of LWU are one month after the minimum and maximum of SWN. The lag, together with the major axis, determines the minor axis of the ellipse. Because the lag is small, the minor axis is small. The small lag of LWU after SWN indicates a small heat capacity of land.

The heat absorbed by the surface is transferred to the atmosphere as sensible and latent heat. The atmosphere is also heated by absorption of shortwave radiance, but the major heating of the lower level is by the surface itself. Figure 14 shows the PC-1 of LWD as a function of PC-1 of LWU. The range of LWD is slightly smaller than the range of LWU. The area within the cycle is very small, i.e. the figure is nearly linear because there is very little lag of LWD behind LWU. 
This very small lag indicates a close coupling of the atmosphere to the land and a small heat capacity of the atmosphere relative to that of land.

The ocean behaves quite differently from land. Figure 15 shows PC-1 for SWN, LWU and LWD as a function of time. LWU lags SWN by about two months, and LWD slightly leads LWU. Figure 16 shows the PC-1 annual cycle of LWU as a function of SWN. Because of the low albedo of ocean compared to land, the range of SWN is slightly greater, i.e. $\pm 70 \mathrm{~W} \mathrm{~m}^{-2}$. The range of LWU is only $\pm 18 \mathrm{~W} \mathrm{~m}^{-2}$, and the maximum and minimum of LWU lags that of SWN by two months. Both of these aspects of the annual cycle of LWU are due to the immense heat capacity of the ocean.

Figure 17 shows the PC-1 annual cycle of LWD plotted against the PC-1 of the annual cycle of LWU for the ocean. The ranges of both variables are very nearly the same, about $\pm 18 \mathrm{~W} \mathrm{~m}^{-2}$, and the figure is nearly linear. As for the land case, the atmosphere is very closely coupled to the ocean. In the first three hodograph plots (Figs. 13, 14, 16), the cycle proceeded counterclockwise, indicating the ordinate lagged the abscissa. In this plot, the annual cycle proceeds in a clockwise direction, so that the LWD slightly leads the LWU for ocean. This shows that the atmosphere with its small thermal mass heats faster in response to the increasing insolation than does the ocean and conversely for decreasing insolation. The atmosphere may be heated by absorption of insolation or by heating of the air over land after which the air moves over the ocean. The latter effect is a small effect, especially in the Southern Hemisphere. The absorption of solar radiation by the atmosphere and the SWN at the ocean surface are both proportional to the top of atmosphere insolation, so that SWN is a rough measure of solar heating of the atmosphere. Because the atmospheric temperature leads that of the ocean surface, the atmosphere (LWD) heats the ocean until two months past the winter solstice, at which time the 
ocean has reached its coolest surface temperature so that LWU is at its minimum. The atmosphere then cools the ocean until two months past the summer solstice.

The ellipse of Fig. 13 suggests a simple model in which the land temperature is driven by SWN and LWU is considered to be a proxy for that surface temperature. The model is assumed to be first order and linear, i.e. of the form

$$
\tau \frac{d y}{d t}+y=f
$$

where $\tau$ is the response time for the process, $t$ is time, and for Fig. 12, $y$ is LWU and $f$ is SWN. The forcing function $\mathrm{SWN}$ is represented as $\exp (i \omega t)$, so the solution of this equation is $\mathrm{y}=(1+i \omega \tau)^{-1} \exp (i \omega t)$

The parameters describing the ellipse of Fig. 13 may be computed as follows. The RMS value of PC-1 for SWN is $45.2 \mathrm{~W} \mathrm{~m}^{-2}$ and for LWU is $44.9 \mathrm{~W} \mathrm{~m}^{-2}$. The RMS is the magnitude of the PC-1 in function space. The inner product of PC-1 for SWN times PC-1 of LWU is computed to be 1879 , which is divided by the product of the RMS of these principal components to give the cosine of the angle between the two functions $(0.926)$. This angle is thus $22.2^{\circ}$, or 0.062 years, the lag of LWU behind SWN. The dashed line in Fig. 13 is the ellipse with these parameters. The ocean response is computed in a similar manner, using a RMS of $49.8 \mathrm{~W} \mathrm{~m}^{-2}$ for SWN PC-1 and $12.4 \mathrm{~W} \mathrm{~m}^{-2}$ for LWU, with a phase lag of LWU of $54.4^{\circ}$ behind SWN. It is represented by the dashed line in Fig. 16.

LWD over the ocean is not considered in this manner because LWD is not the dominant heat source to the ocean. The ocean and atmosphere are both heated by solar radiation, and the LWD leads the ocean temperature because the atmosphere has a much smaller heat capacity and heats faster. 
The description of the relation between the time-dependent response of the LWU and LWD to SWN as first-order systems is quite simple. The description only applies to the time variations and does not include the spatial distribution, nor are the physics addressed. However, the description does apply to $90 \%$ of the variance, indicating that after the results of the complex processes of dynamics and heat transfer have been averaged over the globe, the large picture is simple. The complexity is in the details.

\section{Conclusions}

The NASA/GEWEX Surface Radiation Budget Data Set Release 3.0 has been used to study the annual cycles of longwave surface radiation. The zonal-mean annual-average LWD is found to be 0.87 times the zonal-mean annual-average LWU. The time variations have been studied using a principal component analysis in which land and ocean were treated separately. Over land the first principal components for upward longwave flux LWU and downward longwave flux LWD describe more than $90 \%$ of the variance of the seasonal cycles and are sine waves of one year period. Higher order terms represent semiannual cycles and annual cycles out of phase with the first term. The corresponding empirical orthogonal functions describe the geographical variations and are complex due to the processes in differing climatological regions. The principal components and the EOFs provide a quantitative description of the time and space variations of the surface radiation budget. Over land the RMS of the annual cycle of LWU is $46 \mathrm{~W} \mathrm{~m}^{-2}$, and over ocean it is $13 \mathrm{~W} \mathrm{~m}^{-2}$. Over both land and ocean the RMS of LWN is less than half that of LWU.

The relations between the time-varying LWU and SWN are examined using the PC-1 for each flux, and it is shown that the relation between LWU and SWN can be described as a first- 
order system. For land, LWU lags SWN by $22.2^{\circ}$ or 0.062 year, and for ocean the lag is $54.4^{\circ}$ or 0.15 year.

Acknowledgments: The authors gratefully acknowledge support by the Surface Radiation Budget Program from the NASA Science Mission Directorate through Langley Research Center to Science Systems and Applications Inc. and to the National Institute of Aerospace. They also acknowledge the Atmospheric Science Data Center of Langley Research Center for access to the NASA/GEWEX SRB data set. These data are available at http://eosweb.larc.nasa.gov. 


\section{References}

Bess, T. D., G. L. Smith, T. P. Charlock, and F. G. Rose, 1992: Annual and interannual variations of Earth-emitted radiation based on a 10-year data set. J. Geophys. Res., 97D, $12,825-12,835$.

Bloom., S. and Coauthors, 2005: Documentation and validation of the Goddard Earth Observing System (GEOS) Data Assimilation System--Version 4. NASA Tech. Memo. NASA/TM2005-104606, 187 pp.

Cox, S. J., P. W. Stackhouse, S. K. Gupta, J. C. Mikovitz, and T. Zhang, 2006: The NASA/GEWEX Surface Radiation Budget project: Overview and analysis. Extended Abstracts, $12^{\text {th }}$ Conf. on Atmospheric Radiation, Madison, WI, Amer. Meteor. Soc., 10.1.

Darnell, W. L., W. F. Staylor, S. K. Gupta, and F. M. Denn, 1988: Estimation of surface insolation using Sun-synchronous satellite data. J. Climate, 1, 820-835.

Darnell, W. L., W. F. Staylor, S. K. Gupta, N. A. Ritchey and A. C. Wilber, 1992: Seasonal variation of surface radiation budget derived from International Satellite Cloud Climatology Project C1 data, J. Geophys. Res., 97D, 15,741-15,760.

Fu, Q., K. N. Liou, M. C. Cribb, T. P. Charlock, and A. Grossman, 1997: Multiple scattering parameterization in thermal infrared radiative transfer. J. Atmos. Sci., 54, 2799-2812.

Gupta, S. K., 1989: A parameterization for longwave surface radiation from Sun-synchronous satellite data. J. Climate, 2, 305-320.

Gupta, S. K., W. F. Staylor, W. L. Darnell, A. C. Wilber, and N. A. Ritchey, 1993: Seasonal variation of surface and atmospheric cloud radiative forcing over the globe derived from satellite data. J. Geophys. Res., 98D, 20,761-20,778.

Gupta, S. K., N. A. Ritchey, A. C. Wilber, C. H. Whitlock, G. G. Gibson, and P. W. Stackhouse, 
1999: A climatology of surface radiation budget derived from satellite data., J. Climate, 12, 2691-2710.

Gupta, S. K., P. W. Stackhouse, S. J. Cox, J. C. Mikovitz, and T. Zhang, 2006: Surface Radiation Budget Project completes 22-year data set, GEWEX News, Vol. 16, no. 4, International GEWEX Project Office, Silver Spring, MD, 12-13.

Hatzianastassiou, N., C. Matsukas, D. Hatzidimitriou, C. Pavlakis, M. Drakakis, and I. Vardavas, 2004: Ten year radiation budget of the Earth: 1984-93, Int. J. Climatol., 24, 1785-1802.

Hatzianastassiou, N., C. Matsukas, A. Fotiadi, K. G. Pavlakis, E. Drakakis D. Hatzidimitriou, and I. Vardavas, 2005: Global distribution of Earth's surface shortwave radiation budget, Atmos. Chem. Phys., 5, 2847-2867.

Hsu, C-P. F. and J. M. Wallace, 1976a: The global distribution of the annual and semiannual cycles in precipitation, Mon. Wea. Rev., 104, 1093-1101.

Hsu, C-P. F. and J. M. Wallace, 1976b: The global distribution of the annual and semiannual cycles in sea level pressure, Mon. Wea. Rev., 104, 1597-1601.

Pinker, R., and I. Laszlo, 1992: Modeling surface solar irradiance for satellite applications on a global scale. J. Appl. Meteor., 31, 194-211.

Ramanathan, V., 1986: Scientific use of surface radiation budget data for climate studies. Surface radiation budget for climate applications, NASA RP-1169, pp. 58-86.

Rossow, W. B. and R. A. Schiffer, 1991: ISSCP cloud data products, Bull. Amer. Meteor. Soc., 72, 2-20.

Rossow, W. B., and R. A. Schiffer, 1999: Advances in understanding clouds from ISCCP. Bull. Amer. Meteor. Soc., 80, 2261-2287.

Smith, G. L., D. Rutan, T. P. Charlock, and T. D. Bess, 1990: Annual and interannual variations 
of absorbed solar radiation based on a 10-year data set. J. Geophys. Res., 95D, 16,63916,652 .

Smith, G. L., A. C. Wilber, S. K. Gupta, and P. W. Stackhouse, 2002: Surface radiation budget and climate classification. J. Climate, 15, 1175-1188.

Smith, G. L., P. E. Mlynczak, D. A. Rutan, and T. Wong, 2008: Comparison of the diurnal cycle of outgoing longwave radiation from a climate model with results from ERBE. J. Appl. Meteor. Climatol., 47, 3188-3201.

Stackhouse, P. W., S. K. Gupta, S. J. Cox, J. C. Mikovitz, T. Zhang, and M. Chiacchio, 2004: Twelve-year surface radiation budget data set. GEWEX News, Vol. 14, no. 4, International GEWEX Project Office, Silver Spring, MD, 10-12.

White, G. H. and J. M. Wallace, 1978: The global distribution of the annual and semiannual cycles in surface temperature. Mon. Wea. Rev., 106, 901-906.

Whitlock, C. H. and Coauthors, 1995: First global WCRP shortwave surface radiation budget dataset. Bull. Amer. Meteor. Soc., 76, 905-922.

Wilber, A. C., G. L. Smith, S. K. Gupta and P. W. Stackhouse, 2006: Annual cycles of surface shortwave radiative fluxes. J. Climate, 19, 535-547.

WCRP-69, 1992: Radiation and climate: Report of the fourth session of the WCRP working group on radiative fluxes. WMO/TD-No. 471. 


\section{List of Figures}

FIG. 1. Annual mean map of surface flux $\left(\mathrm{W} \mathrm{m}^{-2}\right)$ for a) LWU, b) LWD, and c) LWN.

FIG. 2. Variation with latitude of the zonal-mean annual-average components of surface SWN, LWU and LWD $\left(\mathrm{W} \mathrm{m}^{-2}\right)$. Also, the dotted line is 0.87 times LWU.

FIG. 3. Variation with latitude of the zonal-mean annual-average $\mathrm{LWN}\left(\mathrm{W} \mathrm{m}^{-2}\right)$.

FIG. 4. First four principal components of the annual cycle of LWU over land in $\mathrm{W} \mathrm{m}^{-2}$.

FIG. 5. First four principal components of the annual cycle of LWD over land in $\mathrm{W} \mathrm{m}^{-2}$.

FIG. 6. First four principal components of the annual cycle of LWN over land in $\mathrm{W} \mathrm{m}^{-2}$.

FIG. 7. First EOF over land for the annual cycle of a) LWU, b) LWD, and c) LWN.

FIG. 8. First four principal components of the annual cycle of LWU over ocean in $\mathrm{W} \mathrm{m}^{-2}$.

FIG. 9. First four principal components of the annual cycle of LWD over ocean in $\mathrm{W} \mathrm{m}^{-2}$.

FIG. 10. First four principal components of the annual cycle of $\mathrm{LWN}$ over ocean in $\mathrm{W} \mathrm{m}^{-2}$.

FIG. 11. First EOF over ocean for the annual cycle of a) LWU, b) LWD, and c) LWN.

FIG. 12. First principal components over land for the annual cycles of SWN, LWU and LWD in $\mathrm{W} \mathrm{m}^{-2}$.

FIG. 13. Monthly-mean values of first principal component of LWU as a function of monthlymean values of first principal component of SWN over land. The dashed line is an ellipse fit to the data.

FIG. 14. Monthly-mean values of first principal component of LWD as a function of monthlymean values of first principal component of LWU over land.

FIG. 15. First principal components over ocean for the annual cycles of SWN, LWU and LWD in $\mathrm{W} \mathrm{m}^{-2}$. 
FIG. 16. Monthly-mean values of first principal component of LWU as a function of monthlymean values of first principal component of SWN over ocean. The dashed line is an ellipse fit to the data.

FIG. 17. Monthly-mean values of first principal component of LWD as a function of monthlymean values of first principal component of LWU over ocean. 
TABLE 1. Annual average surface radiation fluxes and root-mean-square of annual cycles $\left(\mathrm{W} \mathrm{m}^{-2}\right)$.

\begin{tabular}{|c|c|c|c|c|}
\hline & LWU & LWD & LWN & SWN \\
\hline Average & & & & \\
\hline Global & 396.4 & 343.8 & -52.6 & 166.5 \\
\hline Land, 27.9\% & 374.7 & 307.3 & -67.4 & 138.9 \\
\hline Ocean, 65.8\% & 406.0 & 359.7 & -46.4 & 178.8 \\
\hline RMS & & & & \\
\hline Global & 27.5 & 23.0 & 11.4 & 50.2 \\
\hline Land & 46.0 & 35.5 & 19.2 & 47.0 \\
\hline Ocean & 13.3 & 14.4 & 5.7 & 51.4 \\
\hline
\end{tabular}


TABLE 2. The normalized eigenvalues of, or fraction of variance explained by, the first four PCs of the annual cycles over land.

\begin{tabular}{|c|c|c|c|}
\hline EOF & LW Up & LW Down & LW Net \\
\hline 1 & 0.953 & 0.962 & 0.809 \\
\hline 2 & 0.025 & 0.018 & 0.099 \\
\hline 3 & 0.015 & 0.011 & 0.054 \\
\hline 4 & 0.004 & 0.004 & 0.017 \\
\hline $\begin{array}{c}\text { Sum of first } \\
\text { four terms }\end{array}$ & 0.997 & 0.995 & 0.979 \\
\hline
\end{tabular}


TABLE 3. The normalized eigenvalues of, or fraction of variance explained by, the first four PCs of the annual cycles over ocean.

\begin{tabular}{|c|c|c|c|}
\hline EOF & LW Up & LW Down & LW Net \\
\hline 1 & 0.872 & 0.905 & 0.711 \\
\hline 2 & 0.090 & 0.058 & 0.159 \\
\hline 3 & 0.021 & 0.018 & 0.049 \\
\hline 4 & 0.011 & 0.012 & 0.039 \\
\hline $\begin{array}{c}\text { Sum of first } \\
\text { four terms }\end{array}$ & 0.994 & 0.993 & 0.958 \\
\hline
\end{tabular}

